\title{
ARTICLE OPEN \\ Dry release transfer of graphene and few-layer h-BN by utilizing thermoplasticity of polypropylene carbonate
}

\author{
Kei Kinoshita ${ }^{1}$, Rai Moriya (iD) ${ }^{1}$, Momoko Onodera ${ }^{1}$, Yusai Wakafuji ${ }^{1}$, Satoru Masubuchi ${ }^{1}$, Kenji Watanabe $\mathbb{D}^{2}$, Takashi Taniguchi ${ }^{2}$ and
} Tomoki Machida ${ }^{1}$

We demonstrate the dry release transfer of single-layer and bi-layer graphene and few-layer h-BN by utilizing poly(propylene) carbonate (PPC) films. Because of the strong adhesion between PPC and 2D materials around room temperature, we demonstrate that single-layer to few-layer graphene, as well as few-layer h-BN can be fabricated on a spin-coated PPC film/SiO $/ 2 / \mathrm{Si}$ substrate via the mechanical exfoliation method. In addition, we show that these single-layer to few-layer crystals are clearly distinguishable using an optical microscope with the help of optical interference. Because of the thermoplastic properties of PPC film, the adhesion between the $2 \mathrm{D}$ materials and PPC significantly decreases at about $70^{\circ} \mathrm{C}$. Therefore, by tuning the temperature, the graphene and h-BN flakes on PPC can be easily dry-transferred onto another h-BN substrate. We demonstrate the fabrication of encapsulated h$\mathrm{BN} / g$ raphene/h-BN devices and graphene/few-layer h-BN/graphene vertical-tunnel-junction devices using this method. Our finding provides a simple method for constructing high-quality graphene and h-BN-based van der Waals heterostructures with a minimum amount of polymer contamination.

npj 2D Materials and Applications (2019)3:22 ; https://doi.org/10.1038/s41699-019-0104-8

\section{INTRODUCTION}

Up until now, various kinds of van der Waals (vdW) heterostructures composed of graphene, hexagonal boron nitridee (h-BN), and other two-dimensional (2D) materials have been fabricated for the purposes of fundamental research and applications. ${ }^{1,2}$ To construct these heterostructures, various transfer methods for 2D materials have been explored, ${ }^{3}$ including the (1) wet release transfer, ${ }^{4}$ (2) dry release transfer, ${ }^{5-7}$ and (3) stamping methods. ${ }^{8-11}$ Among them, the dry release transfer method has been recognized as the most versatile and flexible technique to explore various kinds of heterostructures. In this method, flakes of the 2D material are first fabricated on a polymer sheet and are subsequently transferred onto another material (typically, exfoliated h-BN on a Si substrate) without dissolving the polymer sheets by organic solvents. The most commonly used polymer for the dry transfer method is polydimethylpolysiloxane (PDMS). ${ }^{5}$ Various vdW heterostructures have been fabricated based on this method for utilizing transition metal dichalcogenides such as $\mathrm{MoS}_{2}, \mathrm{WSe}_{2}$, and $\mathrm{NbSe}_{2}$, as well as black phosphorus. Examples of the applications of these heterostructures are highquality quantum Hall effect devices, ${ }^{12,13}$ superconducting devices, ${ }^{14-17}$ optoelectronic devices, ${ }^{18-21}$ and spintronics devices. ${ }^{22-24}$ However, the PDMS-based dry transfer method has a limited capability for use in single-layer, bi-layer, and few-layerthick graphene and few-layer-thick h-BN because of the poor adhesion between PDMS and these materials which makes it difficult to fabricate these thin crystals on PDMS sheets, and owing to the poor visibility of the thin flakes prepared on PDMS sheet. ${ }^{5,25-27}$ Alternative polymers that enable reliable exfoliation and dry release transfer of thin graphene and h-BN have been in high demand. In this study, we demonstrate the dry release transfer method using poly(propylene) carbonate (PPC) for fabricating graphene-based and h-BN-based vdW heterostructures. The main advantages of this method are summarized in the following three points. (1) Because of the strong adhesion of PPC to graphene and h-BN at room temperature (RT), appropriately large flakes of thin graphene and h-BN can be obtained with the mechanical exfoliation method. (2) Single-layer graphene, as well as thin $\mathrm{h}-\mathrm{BN}$, fabricated on a PPC/SiO $2 / \mathrm{Si}$ structure is visible under an optical microscope. (3) As the adhesion between PPC and the flakes significantly decreases at high temperatures, we demonstrate the dry release transfer of graphene and h-BN on a h-BN/ $\mathrm{SiO}_{2} / \mathrm{Si}$ substrate at about $70^{\circ} \mathrm{C}$. The proposed dry release transfer method is suitable for fabricating graphene-based and h-BNbased vdW heterostructures.

\section{RESULTS AND DISCUSSION}

First, we show the numerical calculation of optical contrast for graphene and h-BN on different substrates, and the results are presented in Fig. 1. The optical contrast for each substrate was calculated based on the multilayer Fresnel reflection method. ${ }^{28-30}$ We consider the case of visible light incidence from air onto the multi-layer structure including graphene, h-BN, $\mathrm{PPC}, \mathrm{SiO}_{2}$, and $\mathrm{Si}$. The refractive index values of graphene, $\mathrm{PPC}, \mathrm{SiO}_{2}$, and $\mathrm{Si}$ are taken from the literature. ${ }^{31-33}$ We consider the results for graphene/PPC/SiO $2 / S i$ as depicted in Fig. 1a. The single-layer graphene is assumed to have a thickness $d_{1}=0.34 \mathrm{~nm}$ and complex wavelength-independent refractive index $n_{1}(\lambda)=$ 2.6-1.3i. ${ }^{28}$ For multilayer graphene, we use the thickness of $N d_{1}$, where $N$ represents the number of layers. We changed the thickness of the PPC layer $d_{2}$ from 0 to $1000 \mathrm{~nm}$ with a refractive

\footnotetext{
${ }^{1}$ Institute of Industrial Science, University of Tokyo, 4-6-1 Komaba, Meguro, Tokyo 153-8505, Japan and ${ }^{2}$ National Institute for Materials Science, 1-1 Namiki, Tsukuba 305-0044, Japan

Correspondence: Rai Moriya (moriyar@iis.u-tokyo.ac.jp) or Tomoki Machida (tmachida@iis.u-tokyo.ac.jp)
}

Received: 12 January 2019 Accepted: 26 April 2019

Published online: 17 May 2019 
(a)

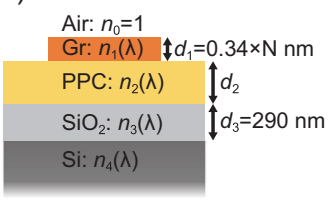

(f)

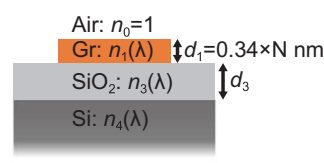

(k)

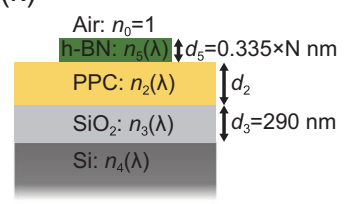

(p)

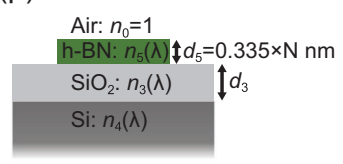

(b)

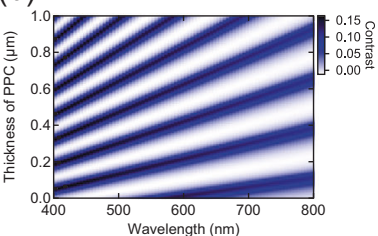

(g)

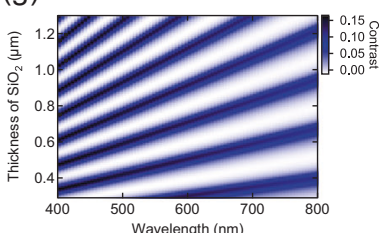

(I)

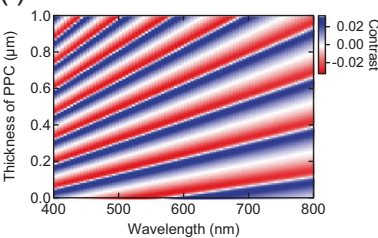

(q)

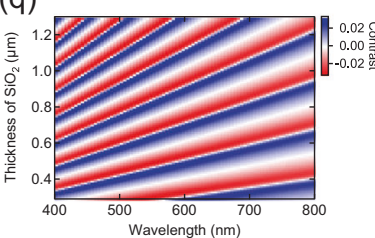

(c)

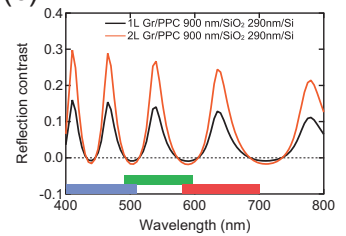

(h)

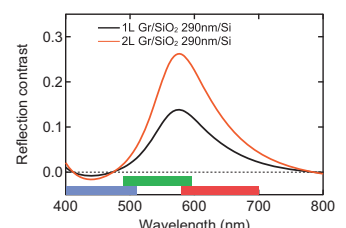

(m)

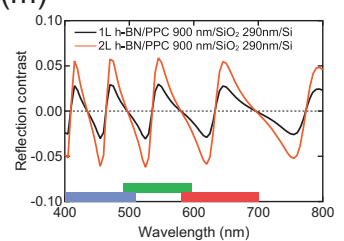

(r)

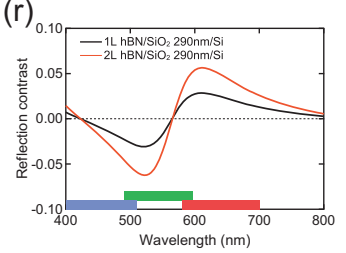

(d)

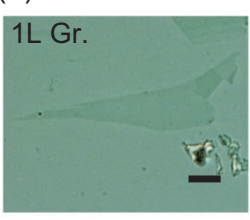

(e)

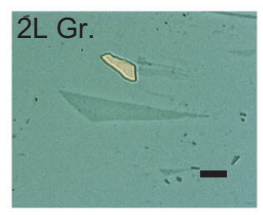

(i)

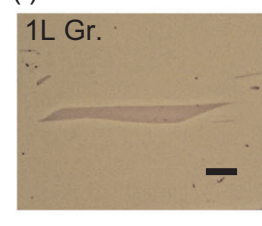

(n)

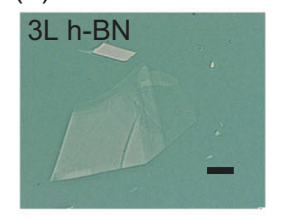

(j)

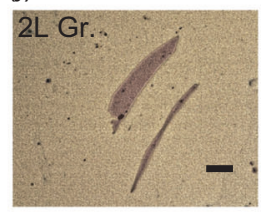

(o)

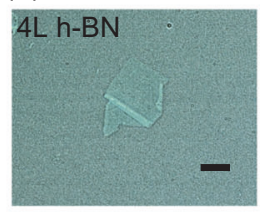

Fig. 1 Schematics, calculated results, and optical micrographs of a-e graphene (Gr)/ poly(propylene) carbonate $(\mathrm{PPC}) / \mathrm{SiO} \mathrm{O}_{2} / \mathrm{Si}, \mathbf{f}-\mathbf{j} \mathrm{Gr} / \mathrm{SiO}{ }_{2} / \mathrm{Si}$, k-o h-BN/PPC/SiO $/ 2 / \mathrm{Si}$, and $\mathbf{p}-\mathbf{r}$ h-BN/SiO $/ 2$ Si structures. a, $\mathbf{f}, \mathbf{k}, \mathbf{p}$ Schematics of the device structure with refractive indexes $n_{1}, n_{2}, n_{3}, n_{4}$, and $n_{5}$ for $\mathrm{Gr}, \mathrm{PPC}, \mathrm{SiO}_{2}, \mathrm{Si}$, and h-BN, respectively. The thicknesses $d_{1}, d_{2}, d_{3}, d_{4}$, and $d_{5}$ are also depicted for $\mathrm{Gr}, \mathrm{PPC}, \mathrm{SiO}, \mathrm{Si}, \mathrm{h}-\mathrm{BN}$, respectively. $\mathbf{b}, \mathbf{g}, \mathbf{I}$, q Calculated optical contrast as a function of wavelength and thickness for $\mathbf{b}, \mathbf{I}$ PPC and $\mathbf{g}, \mathbf{q ~ S i O} 2 . \mathbf{c}, \mathbf{h}, \mathbf{m}, \mathbf{r}$ Representative calculated optical contrast for single-layer and bi-layer $\mathbf{c}, \mathbf{h} \mathrm{Gr}$ and $\mathbf{m}, \mathbf{r} \mathbf{h}-\mathrm{BN}$. $\mathbf{d}, \mathbf{e}, \mathbf{i}, \mathbf{j}, \mathbf{n}, \mathbf{o}$ Optical micrographs of $\mathbf{d}, \mathbf{i}$ single-layer $\mathrm{Gr}, \mathbf{e}, \mathbf{j}$ bi-layer $\mathrm{Gr}$, and $\mathbf{n}, \mathbf{o}$ threelayer and four-layer h-BN. All the scale bars are $10 \mu \mathrm{m}$

index of $n_{2}(\lambda)=1.463$. The thickness of the $\mathrm{SiO}_{2}$ layer $d_{3}$ is fixed at $d_{3}=290 \mathrm{~nm}$ with a wavelength-dependent refractive index $n_{3}(\lambda)$ consisting of only the real part only (typically, $n_{3}(\lambda) \sim 1.458$ for $\lambda$ $\sim 600 \mathrm{~nm}$ ). The thickness of the Si layer is assumed to be semiinfinite with a wavelength-dependent complex refractive index $n_{4}(\lambda)$ (the refractive index data for $\mathrm{Si}$ and $\mathrm{SiO}_{2}$ are provided in the supplementary information). Using the geometry depicted in Fig. $1 \mathrm{a}$, the reflected light intensity can be written as $\left(n_{1}=n_{0}=1\right)$ of graphene:

$C=\frac{I\left(n_{1}=1\right)-I\left(n_{1}\right)}{I\left(n_{1}=1\right)}$.

The results are plotted as a function of the incident light wavelength and PPC thickness, as shown in Fig. 1b. The contrast

$I\left(n_{1}\right)=\left|\frac{e^{i \delta_{3}}\left\{e^{i \delta_{2}}\left(r_{0} e^{i \delta_{1}}+r_{1} e^{-i \delta_{1}}\right)+r_{2} e^{-i \delta_{2}}\left(e^{-i \delta_{1}}+r_{0} r_{1} e^{i \delta_{1}}\right)\right\}+r_{3} e^{-i \delta_{3}}\left\{e^{-i \delta_{2}}\left(e^{-i \delta_{1}}+r_{0} r_{1} e^{i \delta_{1}}\right)+r_{2} e^{i \delta_{2}}\left(r_{0} e^{i \delta_{1}}+r_{1} e^{-i \delta_{1}}\right)\right\}}{e^{i \delta_{3}}\left\{e^{i \delta_{2}}\left(e^{i \delta_{1}}+r_{0} r_{1} e^{-i \delta_{1}}\right)+r_{2} e^{-i \delta_{2}}\left(r_{0} e^{-i \delta_{1}}+r_{1} e^{i \delta_{1}}\right)\right\}+r_{3} e^{-i \delta_{3}}\left\{e^{-i \delta_{2}}\left(r_{0} e^{-i \delta_{1}}+r_{1} e^{i \delta_{1}}\right)+r_{2} e^{i \delta_{2}}\left(e^{i \delta_{1}}+r_{0} r_{1} e^{-i \delta_{1}}\right)\right\}}\right|^{2}$

where

$r_{0}=\frac{n_{0}-n_{1}}{n_{0}+n_{1}}$

$r_{1}=\frac{n_{1}-n_{2}}{n_{1}+n_{2}}$

$r_{2}=\frac{n_{2}-n_{3}}{n_{2}+n_{3}}$

are the reflection coefficients for different interfaces, and $\delta_{1}=$ $2 \pi n_{1} d_{1} / \lambda, \delta_{2}=2 \pi n_{2} d_{2} / \lambda$, and $\delta_{3}=2 \pi n_{3} d_{3} / \lambda$ characterize the phase shifts when light passes through the $n$th layer (detail information of calculation procedure is provided in the supplementary information). Then, the optical contrast $C$ is defined as the relative intensity of reflected light in the presence $\left(n_{1} \neq 1\right)$ and absence curve for single and bi-layer graphene on PPC with a thickness of $900 \mathrm{~nm}$ is presented in Fig. 1c. Contrast exhibits zero to positive values and it depends on wavelength. The graphene-covered region exhibits significant absorption for green, blue, and red light, thus rendering a region on the image that is darker than the surrounding area. This demonstrates that graphene on the PPC/ $\mathrm{SiO}_{2} / \mathrm{Si}$ substrate can be distinguishable with an optical microscope. For comparison, the optical contrast for graphene $/ \mathrm{SiO}_{2} / \mathrm{Si}$, as depicted in Fig. $1 \mathrm{f}$, is calculated. The variation in optical contrast for different thicknesses of $\mathrm{SiO}_{2}$ and wavelengths is plotted in Fig. $1 \mathrm{~g}$, and the results for single-layer and bi-layer graphene on 290nm-thick $\mathrm{SiO}_{2} / \mathrm{Si}$ are presented in Fig. 1h. The calculated optical contrast in Fig. $1 \mathrm{~h}$ is fully consistent with the literature and clear layer-dependent contrast is exhibited. ${ }^{28,29}$ The contrast curves in Fig. $1 \mathrm{~b}, \mathrm{~g}$ are quite similar. This is due to the similarity of the refractive index of PPC to that of $\mathrm{SiO}_{2}$; thus, the spin-coated PPC 
(a)
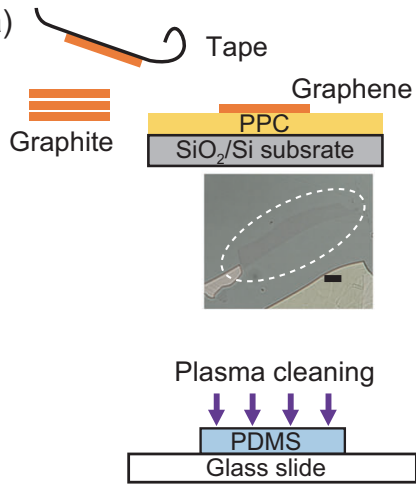

(d)

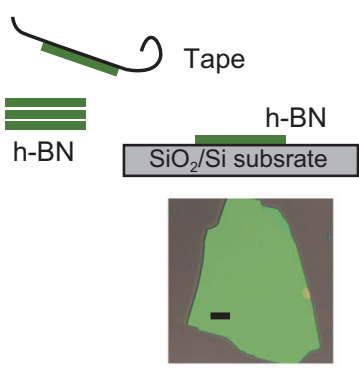

(b)

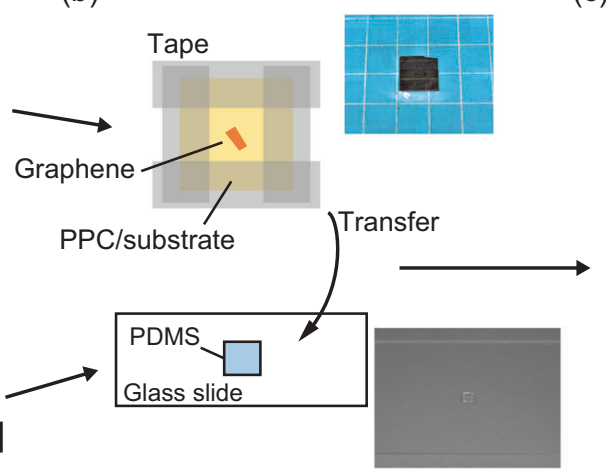

(e)

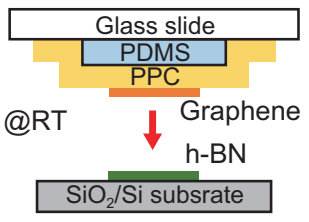

(f)

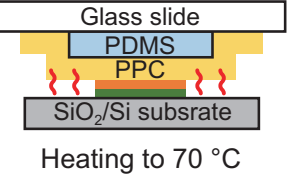

(c)
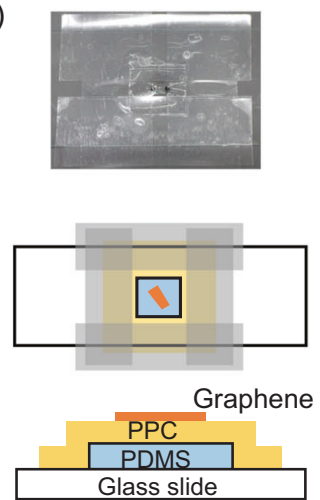

(g)

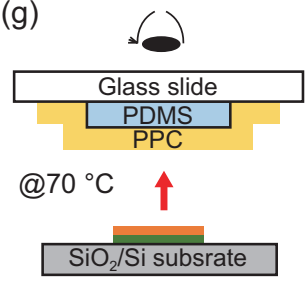

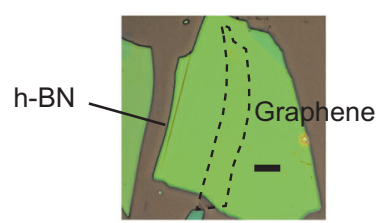

Fig. 2 a-g Schematics of dry transfer fabrication process. a Preparation of graphene (Gr) on $\mathrm{PPC} / \mathrm{SiO}_{2} / \mathrm{Si}$ substrate. An optical micrograph of $\mathrm{Gr} / \mathrm{PPC} / 290-\mathrm{nm}$-thick $\mathrm{SiO}_{2} / \mathrm{Si}$ is also shown. Separately, a piece of polydimethylpolysiloxane (PDMS) is prepared on the glass slide. b Preparation of tape window surrounding graphene area, with its optical micrograph shown in the top panel. Subsequently, this tape window, as well as the Gr/PPC sheet, is transferred onto the PDMS. An optical micrograph of PDMS on the glass slide is shown in the bottom panel. $\boldsymbol{c}$ Prepared Gr/PPC/PDMS structure on glass slide; the optical micrograph is also shown. $\mathbf{d}$ h-BN flake with thickness of about $30 \mathrm{~nm}$ prepared on $\mathrm{SiO}_{2} / \mathrm{Si}$ substrate by mechanical exfoliation technique; the optical micrograph is also shown. e Adjusting the relative positions of Gr and $\mathrm{h}-\mathrm{BN}$ flakes under an optical micrograph and making gentle contact at room temperature. $f$ Heating the stage to $70^{\circ} \mathrm{C}$ while graphene and h-BN are in contact. $g$ Gentle separation of glass slide from $\mathrm{SiO}_{2} / \mathrm{Si}$ substrate; the optical micrograph of the fabricated $\mathrm{Gr} / \mathrm{h}$ - $\mathrm{BN}$ structure on the $\mathrm{SiO}_{2} / \mathrm{Si}$ substrate is also shown. All the scale bars are $10 \mu \mathrm{m}$

on $\mathrm{SiO}_{2} / \mathrm{Si}$ gives rise to a similar effect with the increase in $\mathrm{SiO}_{2}$ thickness in the $\mathrm{SiO}_{2} / \mathrm{Si}$ structure. Therefore, it is a natural consequence that few-layer graphene is visible on a $\mathrm{PPC} / \mathrm{SiO}_{2} / \mathrm{Si}$ substrate with a moderately thin PPC, as studied here. Optical micrographs for single- and bi-layer graphene on 900-nm-thick $\mathrm{PPC} / 290$-nm-thick $\mathrm{SiO}_{2} / \mathrm{Si}$ are shown in Fig. 1d, e, respectively. We find that single- and bi-layer graphene on $\mathrm{PPC} / \mathrm{SiO}_{2} / \mathrm{Si}$ can be visible under an optical microscope. For comparison, optical micrographs of single-layer and bi-layer graphene on 290-nmthick $\mathrm{SiO}_{2} / \mathrm{Si}$ taken with the same optical microscope are also shown in Fig. $1 \mathrm{i}, \mathrm{j}$, respectively. Here, images were taken in highdynamic-range mode to enhance the visibility of the micrograph.

We also calculated the contrast for the h-BN/PPC/290-nm-thick $\mathrm{SiO}_{2} / \mathrm{Si}$ structure, as illustrated in Fig. $1 \mathrm{k}$. Here, the thickness for $\mathrm{h}-$ $\mathrm{BN}$ is assumed to be $d_{5}=0.335 \mathrm{~nm}$ with a wavelengthindependent refractive index of $n_{5}(\lambda)=2.2 .^{34}$ The results are plotted as a function of the incident light wavelength and PPC thickness, as shown in Fig. 1l. The contrast curves for single and bilayer h-BN on PPC with a thickness of $900 \mathrm{~nm}$ are presented in Fig. $1 \mathrm{~m}$. For comparison, the optical contrast for $\mathrm{h}-\mathrm{BN} / \mathrm{SiO}_{2} / \mathrm{Si}$, as depicted in Fig. $1 \mathrm{p}$, is calculated. The variation in optical contrast for different thicknesses of $\mathrm{SiO}_{2}$ and wavelengths is plotted in Fig. $1 \mathrm{q}$, and the results for single-layer and bi-layer h-BN on 290-nmthick $\mathrm{SiO}_{2} / \mathrm{Si}$ are presented in Fig. $1 \mathrm{r}$. For both the $\mathrm{PPC} / \mathrm{SiO}_{2} / \mathrm{Si}$ and $\mathrm{SiO}_{2} / \mathrm{Si}$ substrate, h-BN exhibits a positive or negative optical contrast depending on the wavelength. These positive and negative contrasts could easily cancel out each other under optical microscope observation with white light. Thus, compared to graphene, few-layer $\mathrm{h}-\mathrm{BN}$ is more difficult to distinguish with a microscope. Previous experiments on few-layer h-BN on a $\mathrm{SiO}_{2} / \mathrm{Si}$ substrate used optical filters and optimization of the $\mathrm{SiO}_{2}$ thickness to enhance visibility. ${ }^{34}$ Because of the similarity between Fig. $1 \mathrm{l}, \mathrm{q}$, we believe that few-layer h-BN can be easily distinguishable using filters and by optimizing the thickness of both PPC and $\mathrm{SiO}_{2}$. Nevertheless, we show that even without optimization, the technologically important three-layer and fourlayer h-BN can be distinguishable on a $900-\mathrm{nm}$-thick $\mathrm{PPC} / \mathrm{SiO}_{2} / \mathrm{Si}$ substrate, and their optical micrographs are presented in Fig. 1n, o. These results indicate that the $\mathrm{PPC} / \mathrm{SiO}_{2} / \mathrm{Si}$ structure enables atomically thin $2 \mathrm{D}$ crystals to be exfoliated, and their thickness can be identified with an optical microscope.

Here, we show a method to demonstrate the dry transfer of single-layer graphene on a h-BN substrate. As the thin graphene and $\mathrm{h}-\mathrm{BN}$ are visible on the PPC film, we used this 2D material/PPC stack for the dry transfer of $2 \mathrm{D}$ materials onto another substrate. The detailed transfer procedure for fabricating the graphene/h-BN structure is illustrated in the schematic in Fig. 2. First, the PPC solution was spin-coated on the $\mathrm{SiO}_{2} / \mathrm{Si}$ substrate at a spin-coating speed of $4000 \mathrm{rpm}$; this created a PPC film with a thickness of about $900 \mathrm{~nm}$. Prior to spin coating, the substrate was cleaned by acetone and IPA with ultrasonic agitation. Then, the PPC-coated substrate was baked on a hot plate at $70^{\circ} \mathrm{C}$ for $5 \mathrm{~min}$. The PPC/ $\mathrm{SiO}_{2} / \mathrm{Si}$ substrate was taken away from the hot plate and graphene or h-BN was then deposited on the substrate by the mechanical 


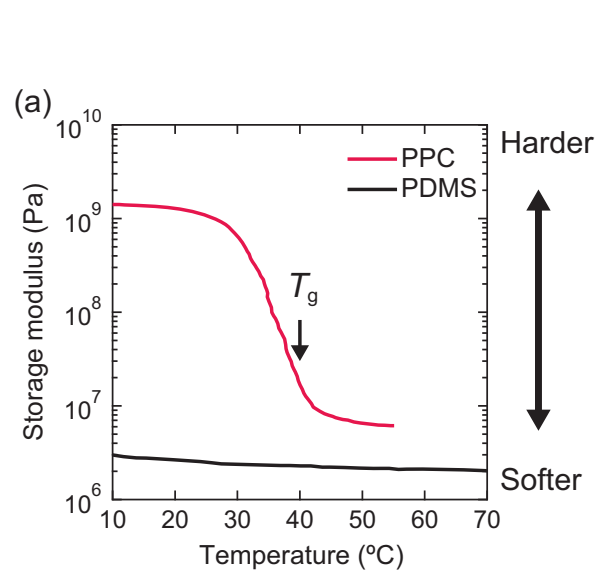

(b)

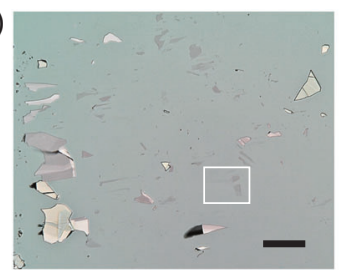

(c)

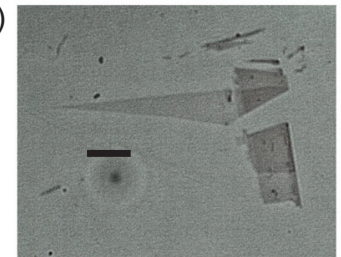

(d)

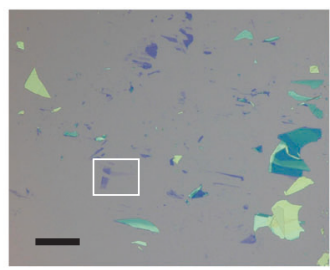

(e)

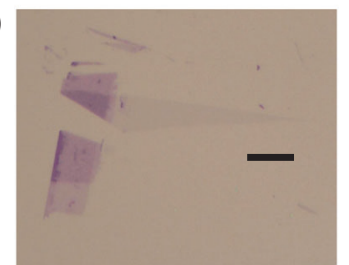

Fig. 3 a Storage modulus as a function of temperature for PPC and PDMS polymers. The data have been obtained from refs., ${ }^{31,38} \mathbf{b}, \mathbf{c}$ Optical micrographs of $\mathrm{Gr}$ and graphite flakes on $900-\mathrm{nm}$-thick PPC/290-nm-thick $\mathrm{SiO}_{2} / \mathrm{Si}$, respectively. Scale bars in b and c are 50 and $10 \mu \mathrm{m}$, respectively. d, e Optical micrographs of transferred $\mathrm{Gr}$ and graphite flakes on 290 -nm-thick $\mathrm{SiO}_{2} / \mathrm{Si}$, respectively. Scale bars in $\mathbf{d}$ and e are 50 and $10 \mu \mathrm{m}$, respectively

exfoliation method at RT, as shown in Fig. 2a. As PPC is known to exhibit a strong adhesion to $2 \mathrm{D}$ materials at around $\mathrm{RT}^{8,10,35,36}$ both few-layer graphene and thick graphite or h-BN with a reasonably large size can be easily fabricated. Separately, a piece of PDMS sheet with a size of approximately $3 \mathrm{~mm}$ (width) $\times 3 \mathrm{~mm}$ (length) $\times 0.4 \mathrm{~mm}$ (depth) was placed on a glass slide. To ensure a strong adhesion between the PDMS and the glass slide, the backside of the PDMS was treated with air plasma for a few minutes. On the PDMS sheet, graphene flakes on the PPC film were transferred from the $\mathrm{SiO}_{2} / \mathrm{Si}$ substrate by the following procedure, as depicted in Fig. 2b, c: (1) prepare the tape window with a size of about $4 \mathrm{~mm} \times 4 \mathrm{~mm}$ on the PPC that surrounds the graphene region; (2) clean the front side of the PDMS sheet with air plasma for a few minutes to ensure a strong adhesion of the PDMS surface; (3) remove the graphene/PPC membrane structures together with the tape by gently removing the tape from the substrate; (4) attach the graphene/PPC membrane on the PDMS sheet by aligning the tape window to the PDMS sheet (Fig. 2C). At the baking temperature of $70^{\circ} \mathrm{C}, \mathrm{PPC}$ can be easily removed from the substrate without using a sacrificed polymer layer between PPC and the substrate. ${ }^{6,7}$ We note that baking with temperature higher than $70^{\circ} \mathrm{C}$, smooth detaching of the PPC sheet from the substrate with this method becomes more difficult. Separately, h$\mathrm{BN}$ flakes were prepared on another $\mathrm{SiO}_{2} / \mathrm{Si}$ substrate by mechanical exfoliation (Fig. 2d). The substrate was pre-cleaned with piranha solution prior to the deposition of h-BN. For transfer, the relative positions of graphene/PPC and h-BN were aligned under optical microscope observation, as shown in Fig. 2e, and the graphene and h-BN were made to gently come into contact each other without heating up the stage. Once they made contact, the substrate stage was heated up to $70^{\circ} \mathrm{C}$ (Fig. 2f). Here, we changed only the setpoint of the substrate heater from RT to $70^{\circ} \mathrm{C}$; then, the substrate heated up with the maximum speed of the heater. When the substrate stage temperature was stable at $70^{\circ} \mathrm{C}$, the substrate and PPC film were slowly separated by moving either the substrate stage or the glass slide stage (Fig. $2 \mathrm{~g}$ ). We found that at temperatures equal to or higher than about $70^{\circ} \mathrm{C}$, the adhesion between graphene and PPC significantly weakens such that graphene can be released from the PPC film and transferred onto h-BN. Thus, the graphene/h-BN structure can be fabricated. Similarly, thick or thin h-BN can be transferred onto the h-BN or graphene. The dry transfer operates within the temperature range of $70-100^{\circ} \mathrm{C}$, depending on the adhesion between PPC and PDMS. The PPC film tends to detach from PDMS during transfer when the substrate temperature is higher than $100^{\circ} \mathrm{C}$. Here, we would note that increasing temperature have dual roles for successively transferring the graphene onto thick $\mathrm{h}-\mathrm{BN}$; one is for easier detaching graphene or thin h-BN from PPC sheet and second is for preventing pick up targeted h-BN from $\mathrm{SiO}_{2} / \mathrm{Si}$ substrate with PPC; both of these are crucial to success our method. The process does not require graphene (or h-BN) to be exposed to organic solvents; thus, this can be regarded as a dry release transfer. To completely remove the PPC residue from the graphene surface, the sample was annealed at $350{ }^{\circ} \mathrm{C}$ in an $\mathrm{Ar} / 3 \%$ $\mathrm{H}_{2}$ atmosphere for $1 \mathrm{~h}$; this temperature is above the decomposition temperature of PPC of about $280^{\circ} \mathrm{C}^{37}$ The air-plasma treatment process during transfer can be replaced with an $\mathrm{O}_{2}$ plasma or an equivalent process to improve the adhesion at the PPC/PDMS and PDMS/glass interfaces. We also note that these plasma treatment processes are not crucial steps to achieve flake transfer but they reduce the risk of PDMS or PPC sheet falling off from the glass slide or PDMS during transfer.

Here, we discuss the mechanism of the PPC-based dry transfer. PPC is known as a thermoplastic material such that it becomes very soft at higher temperatures, whereas it hardens at RT. To compare this to PDMS, we plot the storage modulus of these materials taken from the literature in Fig. $3 a^{31,38}$ The storage modulus represents the mechanical hardness of the material. The glass transition temperature $\left(T_{\mathrm{g}}\right)$ of PPC is about $40^{\circ} \mathrm{C}$. Below this temperature, the storage modulus rapidly increases and saturates at a lower temperature. Here, PPC behaves as a solid and is difficult to deform. The adhesion between the 2D crystal and PPC is known to be strong in this region. In fact, the pick-up of thick h-BN from the $\mathrm{SiO}_{2} / \mathrm{Si}$ substrate with a PPC sheet has been performed around this temperature regime. . $^{80,35,36}$ We demonstrate that reasonably large graphene and h-BN flakes can be easily fabricated with mechanical exfoliation. An optical micrograph of graphene and graphite on PPC fabricated under this condition is presented in Fig. $3 b, c$, respectively. Heating the PPC above about $40^{\circ} \mathrm{C}$ drastically reduces its storage modulus. Here, PPC is glass and becomes soft. The adhesion between the 2D crystal and PPC gradually decreases with the increase in temperature above this region. At high temperatures (about $70^{\circ} \mathrm{C}$ ) where the adhesion between PPC and graphene or PPC and h-BN is sufficiently small, these crystals tend to detach from the PPC and transfer to another substrate. Previous works done by other groups also demonstrated that a h-BN or a h-BN/graphene/h$\mathrm{BN}$ stack can be dry transferred from $\mathrm{PPC}$ sheet to $\mathrm{SiO}_{2} / \mathrm{Si}$ substrate in the temperature range of $70-110^{\circ} \mathrm{C} .{ }^{10,36,39}$ Here, graphene and the graphite flakes respectively shown in Fig. 3b, c are transferred onto the 290-nm-thick $\mathrm{SiO}_{2} / \mathrm{Si}$ substrate using the method explained in Fig. 2, and their optical micrographs are presented in Fig. 3d, e, respectively. Nearly all the graphene and graphite flakes can be 
(a) $1 \mathrm{~L} \mathrm{Gr}$

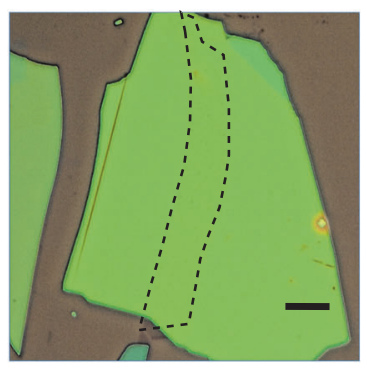

(e)

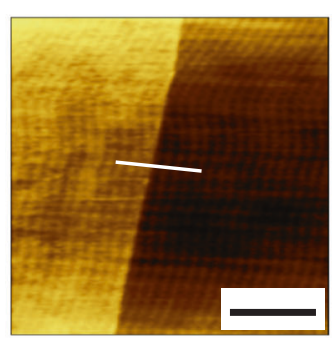

(i)

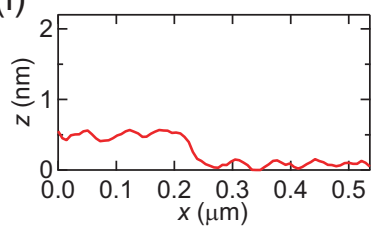

(b) $2 \mathrm{~L} \mathrm{Gr}$

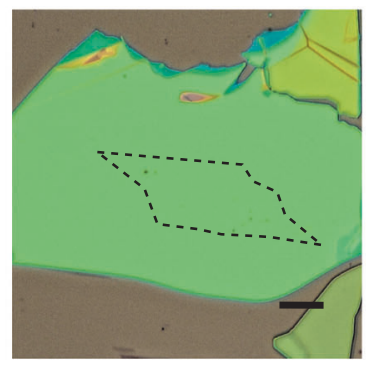

(f)

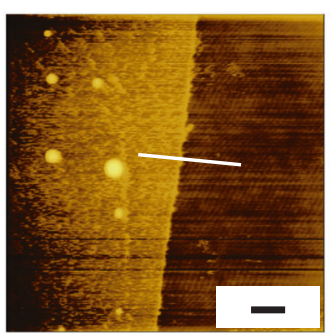

(j)

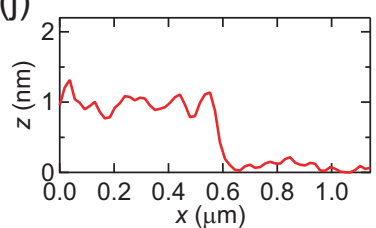

(c) $3 \mathrm{~L} \mathrm{~h}-\mathrm{BN}$

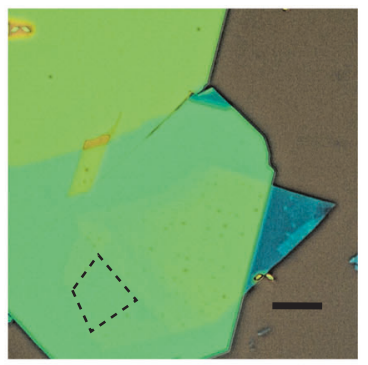

(g)

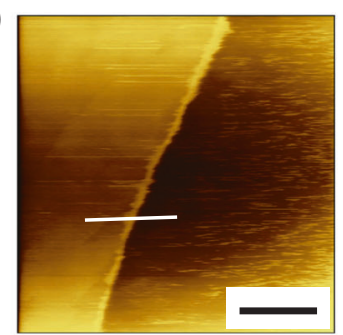

(k)

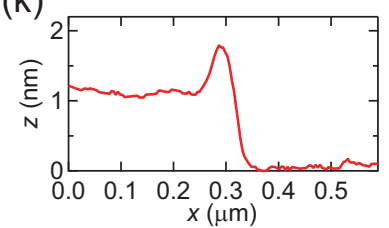

(d) 4L h-BN

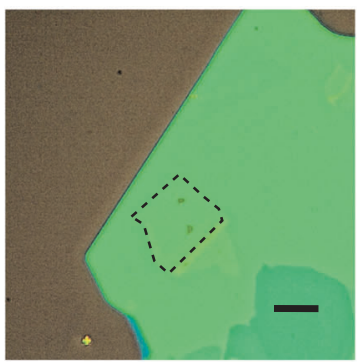

(h)

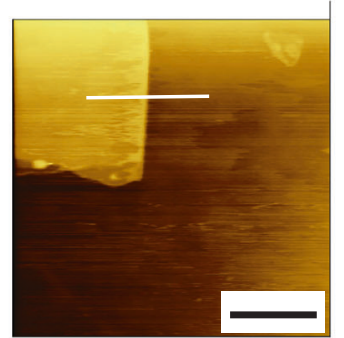

(I)

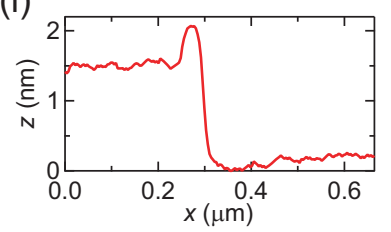

Fig. 4 a-d Optical micrograph, e-h atomic force microscopy (AFM) topographic image, and $\mathbf{i}-\mathbf{I}$ AFM height profiles of a, e, i single-layer Gr on thick h-BN, b, $\mathbf{f}$, j bi-layer Gr on thick h-BN, c, $\mathbf{g}, \mathbf{k}$ three-layer $\mathrm{h}-\mathrm{BN}$ on thick $\mathrm{h}-\mathrm{BN}$, and $\mathbf{d}$, $\mathbf{h}$, I four-layer $\mathrm{h}$-BN on thick $\mathrm{h}-\mathrm{BN}$, respectively. The locations of the displayed AFM height profiles $(\mathbf{i}, \mathbf{j}, \mathbf{k}, \mathbf{I})$ are indicated by white lines in panels $(\mathbf{e}, \mathbf{f}, \mathbf{g}, \mathbf{h})$, respectively. Scale bars in $\mathbf{a}-\mathbf{d}$ and $\mathbf{e}-\mathbf{h}$ are $10 \mu \mathrm{m}$ and $500 \mathrm{~nm}$, respectively

transferred from PPC to the $\mathrm{SiO}_{2}$ substrate. This indicates that our dry transfer method relies on the strong reduction of adhesion between the 2D crystal and PPC membrane at high temperatures. Thus, the flakes on the PPC can be easily transferred even on the $\mathrm{SiO}_{2}$ surface. For comparison, the storage modulus curve for PDMS is also presented in Fig. 3a. As the $T_{\mathrm{g}}$ of PDMS is $-28^{\circ} \mathrm{C}$, PDMS does not show a dramatic change in storage modulus with the increase in temperature and is always in the glass state and soft above RT. Because of this softness, PDMS is very effective for the dry transfer of the flake once the flake has been exfoliated onto the PDMS surface. The drawback of this method is the difficulty of the exfoliation step of the thin graphene and h-BN flakes onto PDMS due to the poor adhesion. The advantage of using the PPC presented here is the strong adhesion at RT, which becomes very weak at higher temperatures.

In Fig. $4 a-d$, optical micrographs of the fabricated single-layer and bi-layer graphene and three-layer and four-layer h-BN on a thick $\mathrm{h}-\mathrm{BN} / \mathrm{SiO}_{2} / \mathrm{Si}$ heterostructure are presented, respectively. Topographic image samples were measured by atomic force microscopy (AFM) and are respectively presented in Fig. 4e-h. From both the optical micrograph and AFM image, it appears that in each case, the surface of the transferred graphene or $h$ $\mathrm{BN}$ is clean, without noticeable polymer residue. There are some bubbles presented in the transferred flakes. We infer that the number of bubbles can be reduced by further optimizing transfer conditions such as substrate temperature. ${ }^{10}$ The thicknesses of the single-layer and bi-layer graphene and three-layer and four-layer h-BN on the thick h-BN in Fig. $4 \mathrm{e}-\mathrm{h}$ were measured and the results are presented in Fig. 4i-I, respectively. The thicknesses are determined to be about 0.5 , $0.9,1.2$, and $1.5 \mathrm{~nm}$, respectively. The thickness difference between the flakes is $0.3-0.4 \mathrm{~nm}$ and they are close to the single-layer thickness of both graphene and h-BN; thus, we think these prove the successful dry transfer of single-layer to fewlayer-thick graphene and $\mathrm{h}-\mathrm{BN}$ with a reasonable control of thickness.

Finally, we show the transport properties of graphene and hBN devices fabricated with our proposed dry transfer method. By repeating the PPC-based dry transfer, encapsulated h-BN/ graphene/h-BN and h-BN/graphene/few-layer h-BN/graphene/hBN structures were fabricated. By opting to use a top h-BN layer with a size smaller than the graphene layer, we allow the edge of the graphene to be not fully covered by the top h-BN layer. This enables us to construct a vdW heterostructure without using a one-dimensional edge-contact scheme. ${ }^{8}$ This greatly simplifies the device fabrication as well as eliminates the requirement of fabricating of a global graphite gate with edge contact, which is difficult. We fabricated both top-contact (Fig. 5a) and edge-contact (Fig. 5b) h-BN/graphene/h-BN devices. In addition to this, a top-contact vertical-tunnel-junction device (Fig. 5c) was fabricated. Optical micrographs of the devices are presented in Fig. $5 d-f$, respectively. Note that top-contact device in Fig. $5 \mathrm{a}$ contains two graphene channels that are prepared by successive transfers: one uses a Si back-gate and the other uses a graphite back-gate. Magnetotransport measurements were performed at $\sim 2.0 \mathrm{~K}$. The resistances of the graphene channels were measured under the sweep of the carrier density $n$ by applying a gate voltage to the doped Si back-gate or graphite back-gate, and the results are presented in Fig. $5 \mathrm{~g}, \mathrm{~h}$, respectively. The capacitance of the $\mathrm{h}-\mathrm{BN} / \mathrm{SiO}_{2}$ dielectric was determined from the quantum Hall effect. The mobilities and charge inhomogeneity (extracted from the width of a charge neutrality point resistance peak) of the two top-contact h-BN/ graphene/h-BN devices were calculated as $\sim 260,000 \mathrm{~cm}^{2} / \mathrm{Vs}$ and 
(a) Top contact device

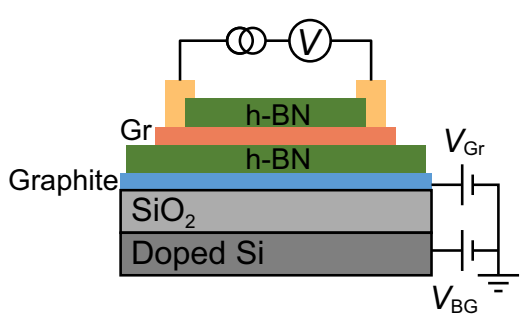

(d)

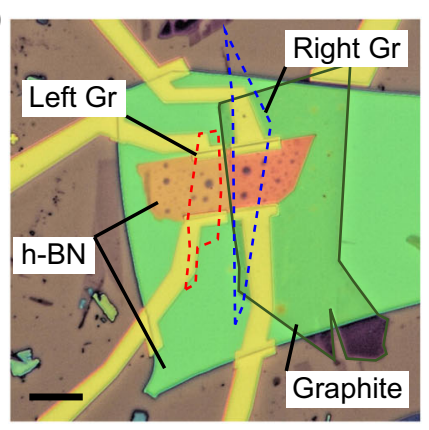

(g)

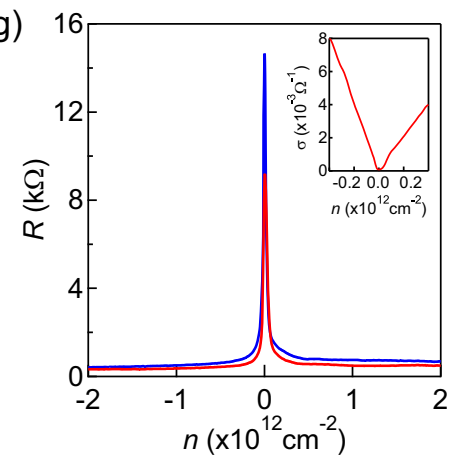

(b) Edge contact device

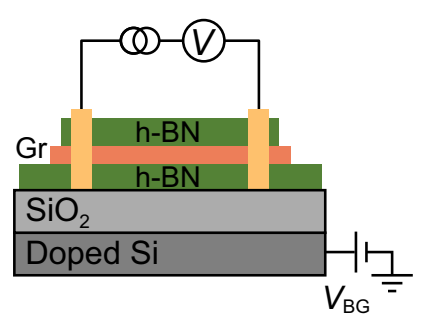

(e)

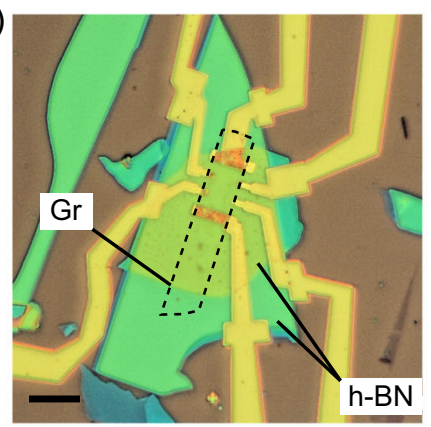

(h)

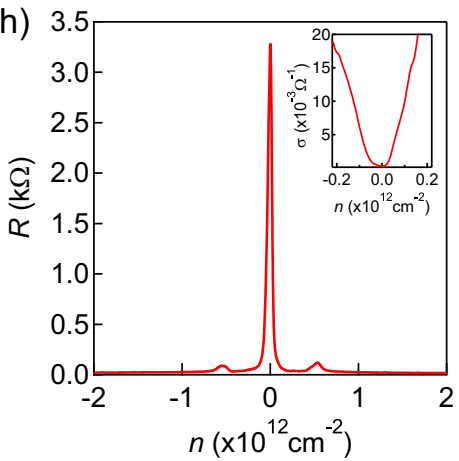

(c) Tunnel junction

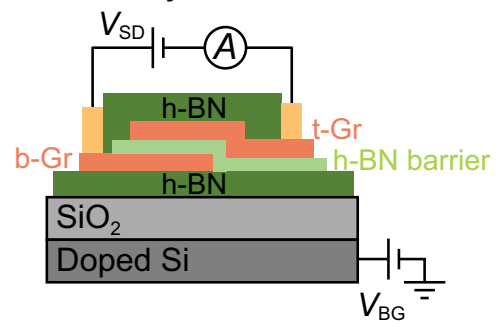

(f)

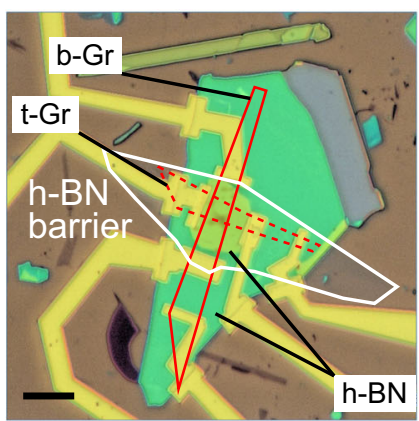

(i)

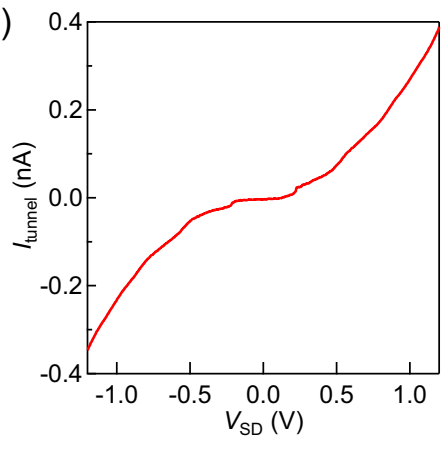

Fig. 5 a-c Schematics of the device structure of a top-contacted $h-B N / G r / h-B N$ sample, $\mathbf{b}$ edge-contacted h-BN/Gr/h-BN sample, and $\mathbf{c}$ h-BN/ $\mathrm{Gr} / \mathrm{few}$-layer $\mathrm{h}-\mathrm{BN} / \mathrm{Gr} / \mathrm{h}-\mathrm{BN}$ vertical-tunnel-junction sample. $\mathbf{d}$, e Photographs of $\mathbf{d}$ top-contact and e edge-contact h-BN/Gr/h-BN devices. Dashed lines outline the location of graphene. Scale bars are $10 \mu \mathrm{m} . \mathbf{g}, \mathbf{h}$ Resistance data as a function of carrier density of Gr for the devices shown in $\mathbf{d}$ and $\mathbf{e}$, respectively; conductivity data is shown in the insets. In $\mathbf{g}$, data shown in red and blue indicates the resistance value of left and right graphene in $\mathbf{d}$, respectively. $\mathbf{f}$ Photograph of $\mathrm{h}-\mathrm{BN} / \mathrm{Gr} / \mathrm{few}$-layer $\mathrm{h}-\mathrm{BN} / \mathrm{Gr} / \mathrm{h}-\mathrm{BN}$ vertical-tunnel-junction device. Solid and dashed red lines indicate the bottom and top graphene, respectively. The white line indicates the thin h-BN tunnel barrier. The thickness of few-layer h-BN is determined via AFM to be about $1.5 \mathrm{~nm}$. Scale bar is $10 \mu \mathrm{m}$. i Current-voltage characteristics of the device shown in $\mathbf{c}$ at $3 \mathrm{~K}$

$\sim 3 \times 10^{10} \mathrm{~cm}^{-2}$ for back-gated device, $\sim 140,000 \mathrm{~cm}^{2} / \mathrm{Vs}$ and $\sim 5 \times 10^{10} \mathrm{~cm}^{-2}$ for graphite-gated device, respectively. These values are comparable to the mobility and the charge inhomogeneity of the edge contact device (Fig. 5h) of $\sim 610,000 \mathrm{~cm}^{2} / \mathrm{Vs}$ and $\sim 5 \times 10^{10} \mathrm{~cm}^{-2}$; thus, we think that quality of the device is not limited by the contact scheme. The extracted mobilities of the h-BN/graphene/h-BN devices approach that of modern h-BN/graphene/h-BN devices; ${ }^{7,8,10,40}$ this indicates the cleanness of our transfer process. This is noticeably high quality considering the fact that the top surface of the transferred graphene flake has been contacted to the PPC polymer during our dry transfer process. This is in contrast to the stamping method $^{8-11}$ where graphene has never contacted to polymer during fabrication. For the top-contacted vertical-tunneljunction device shown in Fig. 5f, we selected an h-BN tunnel barrier with a thickness of about $1.5 \mathrm{~nm}$, as measured by AFM. Current-voltage $(I-V)$ characteristics at about $2.0 \mathrm{~K}$ are presented in Fig. 5i, showing a non-linear change in $/$ with respect to $V$. There are kinks in $I-V$ at around $\pm 0.2 \mathrm{~V}$; we attribute these are due to the tunneling through the localized state within h-BN layer as similar results are published in recent literature based on similar graphene/h-BN/graphene device. ${ }^{41}$ The extracted junction resistance-area product $R A$ is about $1 \times 10^{12} \Omega \mu \mathrm{m}^{2}$; this $R A$ is comparable to that of the h-BN barrier with a similar thickness. ${ }^{42-44}$ These results further prove that the PPC-based dry transfer method can be used to fabricate a multi-stack graphene/h-BN vdW heterostructure with clean interfaces.

In summary, we demonstrate the dry transfer fabrication of graphene as well as h-BN using the PPC-based method. The main advantages of this method are summarized in the following three points: (1) Because of the strong adhesion of PPC to graphene and h-BN at RT, appropriately large flakes of thin graphene and h-BN can be obtained via the mechanical exfoliation method. (2) Single-layer graphene, as well as thin h$\mathrm{BN}$, fabricated on a $\mathrm{PPC} / \mathrm{SiO}_{2} / \mathrm{Si}$ structure is visible under an optical microscope. (3) As the adhesion between the PPC and flakes significantly decreases at high temperatures, we are able to demonstrate the dry release transfer of graphene and h-BN on a h-BN/SiO $2 / S i$ substrate at about $70^{\circ} \mathrm{C}$. Furthermore, we show that the fabricated graphene/h-BN devices exhibit excellent properties. So far, the PDMS-based dry transfer method has been widely used for fabricating TMD-based and black phosphorus-based vdW heterostructures. ${ }^{5}$ With the straightforward addition of a PPC sheet onto the PDMS, we 
demonstrate that graphene as well as few-layer h-BN can be also dry transferred. Our PPC-based dry transfer method is sufficiently simple and enables us to construct the heterostructure using various type of contact scheme. Thus, our results further extend the capability of the dry release transfer method for constructing multilevel high-quality vdW heterostructures.

\section{METHODS}

PPC (Sigma-Aldrich, CAS 25511-85-7) was dissolved into an anisole with a ratio of $15 \%$ PPC: $85 \%$ anisole by weight. The PPC film was prepared by spin-coating the PPC solution onto a 290 -nm-thick $\mathrm{SiO}_{2}$ /highly-doped $\mathrm{Si}$ substrate. The same substrate was used for the mechanical exfoliation of graphene and h-BN. A commercially available PDMS sheet (Gel-Pak PFX4-17 mil.) was used. Graphene was mechanically exfoliated from a graphite crystal (NGS Naturgraphit $\mathrm{GmbH}$ ) using adhesive tape (Nitto Denko, 32B) and was deposited on a PPC-coated $\mathrm{SiO}_{2} / \mathrm{Si}$ substrate or $\mathrm{SiO}_{2} / \mathrm{Si}$ substrate. Using the same method, thin h-BN flakes were exfoliated from its bulk crystal, which was synthesized by the highpressure and high-temperature method and deposited onto the substrate. We chose thick h-BN with thicknesses in the range of 30-50 nm for the substrate and encapsulation for the graphene and thin h-BN. A commercial optical microscope (Keyence, VHX-5000) was used to observe fabricated flakes with a halogen lamp as a light source. To improve the adhesion of the PDMS, a plasma cleaner was operated with air as the plasma source (Harrick Plasma, PDC-32G). The thickness of the flakes was measured with an atomic force microscope (Hitachi High Technologies, SPM400). The device was spin-coated with a PMMA resist, and using electron beam (EB) lithography, electrode patterns were prepared. Au $(\sim 100 \mathrm{~nm}) / \mathrm{Cr}(5 \mathrm{~nm})$ contact electrodes were deposited by EB evaporation. For the edge-contact device, graphene and h-BN were etched using reactive ion etching with a $\mathrm{CF}_{4}$-based gas (Samco, RIE200iP). Immediately after plasma etching, the sample was loaded into the EB evaporation system and the electrodes were deposited. Transport properties were measured in a variable-temperature cryostat equipped with a superconducting magnet that can apply a magnetic field in the out-of-plane direction. After each dry transfer process, the vdW stacks were annealed in a tube furnace under the $0.3 \mathrm{~L} / \mathrm{min}$. of flow of $\mathrm{Ar} / 3 \% \mathrm{H}_{2}$ gas. During the annealing process, temperature setting of the furnace was controlled such that $30 \mathrm{~min}$ for heating up from room temperature to $350^{\circ} \mathrm{C}$, kept at $60 \mathrm{~min}$ at $350^{\circ} \mathrm{C}$, and $30 \mathrm{~min}$ for cooling down from $350^{\circ} \mathrm{C}$ to room temperature.

\section{DATA AVAILABILITY}

The data generated during this study are available from the corresponding author on reasonable request.

\section{ACKNOWLEDGEMENTS}

This work was supported by CREST, Japan Science and Technology Agency (JST) under Grant Number JPMJCR15F3, and by JSPS KAKENHI Grant Numbers JP25107001, JP25107003, JP25107004, JP26248061, JP15K21722, JP15K17433, and JP16H00982.

\section{AUTHOR CONTRIBUTIONS}

K.K., R.M. and T.M. conceived and designed the experiments; K.K. fabricated the devices with the help of R.M., M.O., Y.W. and S.M.; K.W. and T.T. grew the h-BN crystals; K.K performed the device characterization with the help of R.M; R.M., K.K. and T.M. wrote the manuscript using contributions from all authors.

\section{ADDITIONAL INFORMATION}

Supplementary Information accompanies the paper on the npj 2D Materials and Applications website (https://doi.org/10.1038/s41699-019-0104-8).

Competing interests: The authors declare no competing interests.

Publisher's note: Springer Nature remains neutral with regard to jurisdictional claims in published maps and institutional affiliations.

\section{REFERENCES}

1. Geim, A. K. \& Grigorieva, I. V. Van der Waals heterostructures. Nature 499 419-425 (2013).

2. Novoselov, K. S., Mishchenko, A., Carvalho, A. \& Castro Neto, A. H. 2D materials and van der Waals heterostructures. Science 353, aac9439 (2016).

3. Frisenda, R. et al. Recent progress in the assembly of nanodevices and van der Waals heterostructures by deterministic placement of 2D materials. Chem. Soc. Rev. 47, 53-68 (2018).

4. Dean, C. R. et al. Boron nitride substrates for high-quality graphene electronics. Nat. Nanotech. 5, 722-726 (2010).

5. Castellanos-Gomez, A. et al. Deterministic transfer of two-dimensional materials by all-dry viscoelastic stamping. 2D Mater. 1, 011002 (2014).

6. Taychatanapat, T., Watanabe, K., Taniguchi, T. \& Jarillo-Herrero, P. Quantum Hall effect and Landau-level crossing of Dirac fermions in trilayer graphene. Nat. Phys. 7, 621-625 (2011).

7. Kretinin, A. V. et al. Electronic properties of graphene encapsulated with different two-dimensional atomic crystals. Nano Lett. 14, 3270-3276 (2014).

8. Wang, L. et al. One-dimensional electrical contact to a two-dimensional material. Science 342, 614-617 (2013).

9. Zomer, P. J., Guimarães, M. H. D., Brant, J. C., Tombros, N. \& Van Wees, B. J. Fast pick up technique for high quality heterostructures of bilayer graphene and hexagonal boron nitride. Appl. Phys. Lett. 105, 013101 (2014).

10. Pizzocchero, F. et al. The hot pick-up technique for batch assembly of van der Waals heterostructures. Nat. Commun. 7, 11894 (2016).

11. Masubuchi, S. et al. Autonomous robotic searching and assembly of twodimensional crystals to build van der Waals superlattices. Nat. Commun. 9, 1413 (2018).

12. Cui, X. et al. Multi-terminal transport measurements of $\mathrm{MoS}_{2}$ using a van der Waals heterostructure device platform. Nat. Nanotechnol. 10, 534-540 (2015).

13. Li, L. et al. Quantum oscillations in a two-dimensional electron gas in black phosphorus thin films. Nat. Nanotechnol. 10, 608 (2015).

14. Xi, X. et al. Ising pairing in superconducting $\mathrm{NbSe}_{2}$ atomic layers. Nat. Phys. 12, 139-143 (2016).

15. Yabuki, N. et al. Supercurrent in van der Waals Josephson junction. Nat. Commun. 7, 10616 (2016).

16. Kim, M. et al. Strong proximity Josephson coupling in vertically stacked $\mathrm{NbSe}_{2}$-graphene-NbSe 2 van der Waals Junctions. Nano Lett. 17, 6125-6130 (2017).

17. Singh, V. et al. Optomechanical coupling between a multilayer graphene mechanical resonator and a superconducting microwave cavity. Nat. Nanotechnol. 9, 820-824 (2014).

18. Ota, Y. et al. Optical coupling between atomically thin black phosphorus and a two dimensional photonic crystal nanocavity. Appl. Phys. Lett. 110, 223105 (2017).

19. Palacios-Berraquero, C. et al. Large-scale quantum-emitter arrays in atomically thin semiconductors. Nat. Commun. 8, 15093 (2017).

20. Li, D. et al. Two-dimensional non-volatile programmable $p-n$ junctions. Nat. Nanotechnol. 12, 901-906 (2017).

21. Xie, H., Jiang, S., Shan, J. \& Mak, K. F. Valley-selective exciton bistability in a suspended monolayer semiconductor. Nano Lett. 18, 3213-3220 (2018).

22. Lee, J., Wang, Z., Xie, H., Mak, K. F. \& Shan, J. Valley magnetoelectricity in singlelayer MoS. Nat. Mater. 16, 887-891 (2017).

23. Yamasaki, Y. et al. Exfoliation and van der Waals heterostructure assembly of intercalated ferromagnet $\mathrm{Cr}_{1 / 3} \mathrm{TaS}_{2}$. 2D Mater. 4, 041007 (2017).

24. Arai, M. et al. Construction of van der Waals magnetic tunnel junction using ferromagnetic layered dichalcogenide. Appl. Phys. Lett. 107, 103107 (2015).

25. Uwanno, T., Hattori, Y., Taniguchi, T., Watanabe, K. \& Nagashio, K. Fully dry PMMA transfer of graphene on $\mathrm{h}-\mathrm{BN}$ using a heating/cooling system. 2D Mater. 2, 041002 (2015)

26. Goler, S. et al. Self-assembly and electron-beam-induced direct etching of suspended graphene nanostructures. J. Appl. Phys. 110, 064308 (2011).

27. Tien, D. H. et al. Study of graphene-based 2D-heterostructure device fabricated by all-dry transfer process. ACS Appl. Mater. Interfaces 8, 3072-3078 (2016).

28. Blake, P. et al. Making graphene visible. Appl. Phys. Lett. 91, 063124 (2007).

29. $\mathrm{Ni}, \mathrm{Z}$. H. et al. Graphene thickness determination using reflection and contrast spectroscopy. Nano Lett. 7, 2758-2763 (2007).

30. Byrnes, S. J. Multilayer optical calculations. arXiv: 1603.02720 (2016).

31. Luinstra, G. A. \& Borchardt, E. in Synthetic Biodegradable Polymers 29-48 (Springer, Berlin, Heidelberg, 2012).

32. Philipp, H. R. in Handbook of Optical Constants of Solids (ed. Palik, E. D.) 749-763 (Academic Press, Cambridge, 1997).

33. Edwards, D. F. in Handbook of Optical Constants of Solids (ed. Palik, E. D.) 547-569 (Academic Press, Cambridge, 1997).

34. Gorbachev, R. V. et al. Hunting for monolayer boron nitride: optical and Raman signatures. Small 7, 465-468 (2011).

35. Wang, J. I.-J. et al. Electronic transport of encapsulated graphene and $\mathrm{WSe}_{2}$ devices fabricated by pick-up of prepatterned hBN. Nano Lett. 15, 1898-1903 (2015). 
36. Jessen, B. S. et al. Lithographic band structure engineering of graphene. Nat. Nanotechnol. 14, 340-346 (2019).

37. Wang, S. et al. Structure and properties of poly(Propylene Carbonate). Int. J. Polym. Anal. Charact. 3, 131-143 (1997).

38. Barreau, V., Yu, D., Hensel, R. \& Arzt, E. Elevated temperature adhesion of bioinspired polymeric micropatterns to glass. J. Mech. Behav. Biomed. Mater. 76, 110-118 (2017).

39. Larentis, S. et al. Reconfigurable complementary monolayer $\mathrm{MoTe}_{2}$ field-effect transistors for integrated circuits. ACS Nano 11, 4832-4839 (2017).

40. Mayorov, A. S. et al. Micrometer-scale ballistic transport in encapsulated graphene at room temperature. Nano Lett. 11, 2396-2399 (2011).

41. Greenaway, M. T. et al. Tunnel spectroscopy of localised electronic states in hexagonal boron nitride. Commun. Phys. 1, 94 (2018).

42. Britnell, L. et al. Electron tunneling through ultrathin boron nitride crystalline barriers. Nano Lett. 12, 1707-1710 (2012).

43. Kamalakar, M. V., Dankert, A., Bergsten, J., Ive, T. \& Dash, S. P. Enhanced tunnel spin injection into graphene using chemical vapor deposited hexagonal boron nitride. Sci. Rep. 4, 6146 (2014).
44. Yamaguchi, T. et al. Electrical spin injection into graphene through monolayer hexagonal boron nitride. Appl. Phys. Express 6, 073001 (2013).

(C) Open Access This article is licensed under a Creative Commons Attribution 4.0 International License, which permits use, sharing, adaptation, distribution and reproduction in any medium or format, as long as you give appropriate credit to the original author(s) and the source, provide a link to the Creative Commons license, and indicate if changes were made. The images or other third party material in this article are included in the article's Creative Commons license, unless indicated otherwise in a credit line to the material. If material is not included in the article's Creative Commons license and your intended use is not permitted by statutory regulation or exceeds the permitted use, you will need to obtain permission directly from the copyright holder. To view a copy of this license, visit http://creativecommons. org/licenses/by/4.0/.

(c) The Author(s) 2019 